\title{
Chip-based mtDNA mutation screening enables fast and reliable genetic diagnosis of OXPHOS patients
}

Rudy G. E. van Eijsden, $M S c^{1,2}$, Mike Gerards, BSc $c^{2,3}$, Lars M. T. Eijssen, MSc $c^{3,4}$, Alexandra T. M. Hendrickx, BSc ${ }^{1}$, Roselie J. E. Jongbloed, $P h D^{1,2,4}$, John H. J. Wokke, $M D, P h D^{5}$, Rogier Q. Hintzen, $M D, P h D^{6}$, Maria E. Rubio-Gozalbo, $M D, P h D^{7}$, Irenaeus F. M. De Coo, $M D, P h D^{6}$, Egill Briem, $M S c^{8}$, Valeria Tiranti, $P h D^{8}$, and Hubert J. M. Smeets, $P h D^{1,2,3}$

\begin{abstract}
Purpose: Oxidative phosphorylation is under dual genetic control of the nuclear and the mitochondrial DNA (mtDNA). Oxidative phosphorylation disorders are clinically and genetically heterogeneous, which makes it difficult to determine the genetic defect, and symptom-based protocols which link clinical symptoms directly to a specific gene or mtDNA mutation are falling short. Moreover, approximately $25 \%$ of the pediatric patients with oxidative phosphorylation disorders is estimated to have mutations in the mtDNA and a standard screening approach for common mutations and deletions will only explain part of these cases. Therefore, we tested a new CHIP-based screening method for the mtDNA. Methods: MitoChip (Affymetrix) resequencing was performed on three test samples and on 28 patient samples. Results: Call rates were 94\% on average and heteroplasmy detection levels varied from 5-50\%. A genetic diagnosis can be made in almost one-quarter of the patients at a potential output of 8 complete mtDNA sequences every 4 days. Moreover, a number of potentially pathogenic unclassified variants (UV) were detected. Conclusions: The availability of long-range PCR protocols and the predominance of single nucleotide substitutions in the mtDNA make the resequencing CHIP a very fast and reliable method to screen the complete mtDNA for mutations. Genet Med 2006:8(10):620-627.
\end{abstract}

Key Words: mtDNA, resequencing, OXPHOS disease, heteroplasmy, MitoChip

Oxidative phosphorylation (OXPHOS) disorders affect at least 1 in 8,000 of the general population and belong to the group of most common inherited metabolic diseases. ${ }^{1}$ Disease manifestations due to OXPHOS defects can be highly variable, but usually involve tissues with a high energy demand like heart, muscle, renal and the endocrine systems. ${ }^{2}$ Several welldescribed syndromes are known, like Kearns-Sayre (KSS) and Pearson syndrome, Neuropathy Ataxia Retinitis Pigmentosa (NARP), Mitochondrial Encephalomyopathy, Lactic Acidosis and Stroke-like episodes (MELAS) and Myoclonus Epilepsy and Ragged Red Fibers (MERRF). However, OXPHOS defects

\footnotetext{
From the Department of ${ }^{1}$ Clinical Genetics, ${ }^{2}$ Research Institute Growth and Development (GROW), ${ }^{3}$ Department of Population Genetics, Genomics and Bioinformatics, and the ${ }^{4}$ Cardiovascular Research Institute Maastricht (CARIM), Maastricht University, Maastricht, The Netherlands; ${ }^{5}$ Department of Neurology, Rudolf Magnus Institute of Neuroscience, University Medical Centre Utrecht, Utrecht, The Netherlands; ' Department of Neurology, Erasmus MC, Rotterdam, The Netherlands; ${ }^{7}$ Department of Paediatrics and Laboratory Genetic Metabolic Diseases, Maastricht University Hospital, Maastricht, The Netherlands; and the ${ }^{8}$ Pierfranco and Luisa Mariani Centre for the Study of Children's Mitochondrial Disorders - National Neurological Institute "C. Besta," Unit of Molecular Neurogenetics, Milan, Italy.

Dr. H. J. M. Smeets, Maastricht University, Department of Genetics and Cell Biology, P.O. Box 616, 6200 MD, Maastricht, The Netherlands.

Submitted for publication May 2, 2006.
}

Accepted for publication July 5, 2006.

RGEvE and MG contributed equally to this work.

DOI: 10.1097/01.gim.0000237782.94878.05 can also manifest with more common and less specific symptoms, like type 2 diabetes, deafness, encephalopathy, myopathy, and cardiomyopathy. OXPHOS disorders therefore cause significant morbidity and mortality and have a broad impact on public health.

Approximately 25\% of the pediatric patients with OXPHOS disorders have mutations in the mtDNA, but these are difficult to find due to the genetic and clinical heterogeneity. ${ }^{3}$ As the number of mtDNA mutations has increased to over 250 and the clinical specificity lags behind, symptom-based protocols fall short and screening of the entire mtDNA is preferable. ${ }^{4}$ The recently introduced MitoChip (Affymetrix) is a new method for mtDNA resequencing. ${ }^{5,6}$ In this paper we describe our experience of the complete mtDNA screening of 28 patients with OXPHOS disease by MitoChip resequencing.

\section{MATERIALS AND METHODS}

\section{Patient and test samples}

Genomic DNA was isolated from muscle according to the protocol of Mullenbach et al. ${ }^{7}$ DNA from 3 conventionally sequenced patients, a patient with a heteroplasmic 5 bp deletion, ${ }^{8}$ and 28 patients with a clinical and/or biochemical phenotype of OXPHOS disease were analyzed on the MitoChip. These patients were all negative for the MELAS m.3243A $>$ G, MERRF $\mathrm{m} .8344 \mathrm{~A}>\mathrm{G}$, and NARP m.8993T $>\mathrm{C} / \mathrm{G}$ mutations, and for large deletions of the mtDNA. 


\section{MitoChip and experimental procedure}

The MitoChip contains both sense and antisense probes specific for the forward and reverse sequence of two fragments of the mtDNA. One fragment interrogates 15,446 bases of the mtDNA minus the D-loop sequence. The other fragment is a duplicate of the first fragment, comprising 12,938 bases of the mtDNA coding sequence minus the sequence of the $12 \mathrm{~S}$ and $16 \mathrm{~S}$ mitochondrial ribosomal RNA genes. The two fragments are separated on the chip by a control fragment (Affymetrix). Oligonucleotide probes are synthesized in situ on the chip by Affymetrix by standard photolithography and solid-phase DNA synthesis. ${ }^{9,10}$ For each position of the mtDNA fragments, four 25-mer probes are represented on the chip, each with a different nucleotide in the middle (A, G, C, or T) allowing for the detection of all possible nucleotide substitutions. The probe with the correct corresponding nucleotide in the middle for each mtDNA position will give the highest signal intensity after hybridization and scanning. The entire mtDNA was amplified using the Expand Long Template system (Roche) in 2 fragments (A and B) of 8,466 bp and 7,866 bp in length, with an overlap of $235 \mathrm{bp}$. The primers for fragment A were forward primer [5'-ccgcttctggccacagcacttaaacacatc- $\left.3^{\prime}\right]$ and reverse primer [ $5^{\prime}$-aggaggttagttgtggcaat- $\left.3^{\prime}\right]$, and for fragment $\mathrm{B}$ forward primer $\left[5^{\prime}\right.$-gcttcattcattgcccccac- $\left.3^{\prime}\right]$ and reverse primer [5' -ggaggatggtggtcaagggacccctatctg- $\left.3^{\prime}\right]$. The $7.5-\mathrm{kb}$ control sequence was amplified using the primers and template from the Customseq ${ }^{\mathrm{TM}}$ control kit (Affymetrix). PCR products were purified using the QIAQuick PCR cleanup kit (QIAGEN). Equimolar amounts of the amplified fragments $\mathrm{A}$ and $\mathrm{B}$ were pooled and fragmented together with the amplified 7.5-kb control sequence, labeled and hybridized on a pre-hybridized MitoChip as described in the Affymetrix CustomSeq Resequencing protocol. Chips were washed and stained on the GeneChip fluidics station 400 (Affymetrix) using the preprogrammed CustumSeq Resequencing wash and stain protocol (DNA ARRAY-WS2). The MitoChips were scanned using the Affymetrix GeneChip scanner 3000 creating.CEL files for subsequent analysis.

\section{Data analysis}

Affymetrix GeneChip DNA Analysis Software (GDAS) version 3.0.1.3 beta was used to analyze the .CEL files, using the default program settings. A report file was created by GDAS listing the nucleotide variations for both chip fragments compared to the Revised Cambridge Reference Sequence (RCRS). ${ }^{4,11}$ Discrepancies between nucleotide calls for both chip fragments were evaluated manually. The final base changes were written to a text file ("affy output file"). A second analysis was performed using $\mathrm{R}$, a free software environment for statistical computing and graphics. ${ }^{12}$ In the R analysis, each chip (.CEL file) was analyzed separately. For each position the nucleotide with the highest signal intensity was determined, not taking into account the background signal. Nucleotide changes were printed to a text file ("R output file"). Finally the "affy output files" and the "R output files" were compared and combined, resulting in a list of variations for each sample. Discrepancies between the GDAS and R output were evaluated manually by directly looking at the chip image or by conventional sequencing. Unless otherwise mentioned, throughout this paper the phrase "MitoChip analysis" refers to the combined GDAS and R analysis.

\section{Validation of variations and heteroplasmy levels}

Both strands of a fragment carrying a variant were cycle sequenced using the BigDye Terminator v3.1 Cycle sequencing kit, an ABI-PRISM 3100 genetic analyser and the Sequence Analysis 3.7 software package. The level of heteroplasmy was determined by mutation specific restriction digestion. In case a restriction site was gained or lost, fragments were amplified using primers surrounding the underlying variation, otherwise a specific mismatch primer was designed in order to create a restriction site. On PCR amplification, a labeled primer was added to the reaction in the last PCR cycle to label the PCR products. Labeled PCR products were digested and the fragments were analyzed on an ABI-PRISM 3100 genetic analyser using the GeneScan Analysis 3.7 software package (Applied Biosystems). The level of heteroplasmy was determined by calculating the ratio of the mutant or wild type peak area (depending on the gain or loss of a restriction site by the nucleotide variation) and the sum of the mutant and wild type peak areas. Primer sequences and reaction conditions are available on request.

\section{RESULTS}

\section{MitoChip performance and validation}

Based on a total of 32 DNA samples, the average GDAS call rate was $94.0 \%$, ranging from $88.9-96.8 \%$. Of the GDAS "no call" signals, $0.72 \%(212 / 29,366)$ of the chip positions gave a "no call" for each of the 32 chips analyzed, corresponding to $134 \mathrm{mtDNA}$ nucleotide positions. A GDAS call was always given for each of the 32 chips for $12200 \mathrm{mtDNA}$ nucleotide position (20,486 out of 29,366 (70\%) chip positions). For 7 CHIPs GDAS and R analysis were compared for the entire sequence and $\mathrm{R}$ analysis accounted for an average of $24.2 \%$ (ranging from 13-37\%) of the total amount of base changes in the output files. Five unclassified variants (UVs) were identified by the R analysis, which were not detected by the standard GDAS analysis. For the three test samples, which were also sequenced conventionally, 34/35 (97\%) variations were called correctly on the MitoChip. In two test samples, one and six variations were additionally detected by the MitoChip. By reinterpretation of the conventional sequencing results, one of the variations which were detected additionally by the MitoChip proved to be a low percentage heteroplasmic substitution (false negative), and the other additional variants appeared to be falsely detected as a nucleotide variant by the MitoChip (false positives). A known 5-bp deletion was also tested, but not detected. Two DNA samples differing at 16 positions in their mtDNA sequence were mixed at ratios of 1:1, 1:9, and 1:19. In the mix sample analyses (only GDAS), the Total Qual- 
ity Threshold Score setting was set at 30 and 75 . Fifteen of 16 positions were called correctly at a heteroplasmy level of $50 \%$ for both TQT settings. Only one position was called correctly as heteroplasmic in all three mix samples at both TQT settings. At a TQT value of 30, "no call" signals were 3 times more often observed at the 16 investigated heteroplasmy positions than at a TQT value of 75 .

\section{mtDNA variations in 28 patients}

A total of 520 variations were detected in 28 patients, comprising 197 unique nucleotide substitutions. Fifteen heteroplasmic variations were detected in a total of 11 patients. Four of these variations were confirmed by sequencing and mutation specific restriction digestion $(\mathrm{m} .15939 \mathrm{C}>\mathrm{T}$ at $7 \%$, m.13513G $>$ A at $14 \%, \mathrm{~m} .3243 \mathrm{~A}>\mathrm{T}$ at $34 \%$, and $\mathrm{m} .13042 \mathrm{G}>\mathrm{A}$ at $84 \%$ ); two proved to be homoplasmic single base pair insertions (m.3229_3230insA and m.3158_3159insT); two appeared to be false positive; one was shown to be a homoplasmic polymorphism (m.15452C $>$ A); the remaining six were not tested because they were considered not to be pathogenic. Of all detected variations, three were known pathogenic mutations (Table 1), 114 have been reported before as polymorphisms (Supplementary Table 1), 41 did not result in an amino acid change and were most likely polymorphisms (Supplementary Table 1), and 39 were unclassified variants (UVs), some of which were likely pathogenic (Table 2). Eight variants were located in tRNA molecules (Fig. 1). In three patients known pathogenic mutations were detected and in 23 patients UVs were detected. In five patients only polymorphisms were detected.

UVs were evaluated for evolutionary conservation, for functional significance by determining the effect on the tRNA or on the protein, and, when available, for segregation in the family. Seven UVs were located in six tRNA genes (Fig. 1), 11 UVs in the $12 \mathrm{~S}$ and $16 \mathrm{~S}$ rRNA genes, one UV in the mtDNA transcription terminator site, and 20 UVs in the protein encoding genes. Of the eight tRNA variations (Fig. 1), the m.15939C $>$ T variation in the tRNA-Thr gene was heteroplasmic with a mutation load of $7 \%$. The other tRNA variations were all homoplasmic or close to homoplasmy ( $>98 \%$ mutation load). Two of the variations in the protein encoding genes were heteroplasmic $84 \%$ and $70 \%$, respectively, for the $\mathrm{m} .13042 \mathrm{G}>\mathrm{A}$ in the ND5 gene and $\mathrm{m} .14258 \mathrm{G}>\mathrm{A}$ in the ND6 gene.

Single nucleotide insertions were detected as well. A heteroplasmic m.3158A $>$ T transition on the CHIP proved to be a single nucleotide insertion (m.3158_3159ins T) by standard sequencing. Additionally, the homoplasmic m.3229_3230insA insertion was initially detected by the MitoChip analysis as a heteroplasmic m.3229T $>$ A transition.

Table 1

Pathogenic mutations

\begin{tabular}{|c|c|c|c|c|c|c|c|c|c|}
\hline Position & nt change & aa change & Mutation load $^{a}$ & Locus & Disease $^{b}$ & $\begin{array}{c}\text { Number of } \\
\text { samples }\end{array}$ & aa/nt Conservation ${ }^{c}$ & $\begin{array}{l}\text { No. patients } \\
\text { previously } \\
\text { described }\end{array}$ & Reference(s) \\
\hline \multicolumn{10}{|c|}{ Evident pathogenic mutations } \\
\hline 3243 & $\mathrm{~A}>\mathrm{T}$ & - & $34 \%$ & tRNA-Leul & $\mathrm{MM}$ & 1 & 30/31 species & 1 & $\begin{array}{l}\text { Shaag et al. } \\
(1997)^{19}\end{array}$ \\
\hline 3697 & $\mathrm{G}>\mathrm{A}$ & G-S & $>97 \%$ & ND1 & MELAS & 1 & $\begin{array}{l}\mathrm{Hs} / \mathrm{Pt} / \mathrm{Cf} / \mathrm{Mm} / \mathrm{Rn} / \\
\mathrm{Gg} / \mathrm{Dm} / \mathrm{Ag} / \mathrm{Ce} / \\
\mathrm{At} / \mathrm{Os}\end{array}$ & 1 & $\begin{array}{l}\text { Kirby et al. } \\
\qquad(2004)^{21}\end{array}$ \\
\hline \multirow[t]{6}{*}{13513} & $\mathrm{G}>\mathrm{A}$ & $\mathrm{D}-\mathrm{N}$ & $13-15 \%$ & ND5 & $\begin{array}{l}\text { MELAS/Leigh } \\
\text { Disease }\end{array}$ & 1 & $\begin{array}{l}\mathrm{Hs} / \mathrm{Pt} / \mathrm{Cf} / \mathrm{Mm} / \mathrm{Rn} / \\
\mathrm{Gg} / \mathrm{Dm} / \mathrm{Ag} / \mathrm{Ce} / \\
\mathrm{At} / \mathrm{Os}\end{array}$ & 1 & $\begin{array}{l}\text { Santorelli et al. } \\
(1997)^{26}\end{array}$ \\
\hline & & & & & & & & 4 & $\begin{array}{l}\text { Pulkes et al. } \\
\qquad(1999)^{23}\end{array}$ \\
\hline & & & & & & & & 1 & $\begin{array}{l}\text { Penisson- } \\
\text { Besnier et al. } \\
(2000)^{28}\end{array}$ \\
\hline & & & & & & & & 3 & $\begin{array}{l}\text { Chol et al. } \\
\qquad(2003)^{27}\end{array}$ \\
\hline & & & & & & & & 3 & $\begin{array}{l}\text { Kirby et al. } \\
(2003)^{25}\end{array}$ \\
\hline & & & & & & & & 6 & $\begin{array}{l}\text { Sudo et al. } \\
\qquad(2004)^{24}\end{array}$ \\
\hline
\end{tabular}

\footnotetext{
${ }^{a}$ Mutation loads are determined by mutation specific restriction digestion analysis followed by GeneScan analysis.

${ }^{b} \mathrm{MM}$, Mitochondrial Myopathy; MELAS, Mitochondrial Encephalomyopathy, Lactic Acidosis, and Stroke-like episodes.

${ }^{c}$ For the tRNA variations, the nucleotide conservation is shown. For the variations in protein encoding genes, the amino acid conservation is displayed.

Hs, Homo sapiens; Pt, Pan troglodytes; Cf, Canis familiaris; Mm, Mus musculus; Rn, Rattus norvegicus; Gg, Gallus gallus; Dm, Drosophila melanogaster; Ag, Anopheles gambiae; Ce, Caenorhabditis elegans; At, Arabidopsis thaliana; Os, Oryza sativa.

tRNA conservations were taken from the website dealing with the compilation of mammalian tRNA genes (http://mamit-trna.u-strasbg.fr/index.html). ${ }^{32}$
} 
Table 2

Unclassified variants

\begin{tabular}{|c|c|c|c|c|c|c|c|}
\hline Position & nt change & aa change & Mutation load $^{a}$ & Locus & No. of samples & nt Conservation $^{d}$ & Pathogenicity score $^{e}$ \\
\hline \multicolumn{8}{|c|}{ Non-protein-coding region, unclassified variants } \\
\hline 892 & $A>T$ & - & $\mathrm{h}$ & $12 \mathrm{~S}$ rRNA & 3 & - & \\
\hline 1860 & $A>G$ & - & $100 \%^{b}$ & $16 \mathrm{~S}$ rRNA & 1 & - & \\
\hline 2098 & $\mathrm{G}>\mathrm{A}$ & - & $100 \%$ and & $16 \mathrm{~S}$ rRNA & 1 & - & \\
\hline 2259 & $\mathrm{C}>\mathrm{T}$ & - & $100 \%{ }^{b}$ & $16 \mathrm{~S}$ rRNA & 1 & - & \\
\hline 2361 & $\mathrm{G}>\mathrm{A}$ & - & $100 \%{ }^{b}$ & $16 \mathrm{~S}$ rRNA & 1 & - & \\
\hline 2581 & $\mathrm{~A}>\mathrm{G}$ & - & $100 \%^{b}$ & $16 \mathrm{~S}$ rRNA & 1 & - & \\
\hline 2757 & $\mathrm{~A}>\mathrm{G}$ & - & $100 \%^{b}$ & 16S rRNA & 1 & - & \\
\hline 2768 & $\mathrm{~A}>\mathrm{G}$ & - & $100 \%^{b}$ & $16 \mathrm{~S}$ rRNA & 2 & - & \\
\hline 2825 & $\mathrm{G}>\mathrm{C}$ & - & $\mathrm{h}$ & 16S rRNA & 4 & - & \\
\hline 3105 & $\mathrm{~A}>\mathrm{G}$ & - & $100 \%^{b}$ & $16 \mathrm{~S}$ rRNA & 1 & - & \\
\hline 3159 & ins T & - & $100 \%^{b}$ & $16 \mathrm{~S}$ rRNA & 1 & - & \\
\hline 3229 & insA & - & $\mathrm{h}$ & $\begin{array}{r}\text { Transcription } \\
\text { terminator }\end{array}$ & 1 & - & \\
\hline 4336 & $\mathrm{~T}>\mathrm{C}$ & - & $>98 \%$ & tRNA-Gln & 2 & $13 / 31$ species & \\
\hline 5558 & $\mathrm{~A}>\mathrm{G}$ & - & $100 \%$ & tRNA-Trp & 1 & $31 / 31$ species & \\
\hline 5592 & $\mathrm{~A}>\mathrm{G}$ & - & $100 \%$ & tRNA-Ala & 1 & $28 / 31$ species & \\
\hline 5850 & $\mathrm{~T}>\mathrm{C}$ & - & $>98 \%$ & tRNA-Tyr & 1 & $30 / 31$ species & \\
\hline 12308 & $\mathrm{~A}>\mathrm{G}$ & - & $100 \%^{b}$ & tRNA-Leu2 & 5 & $31 / 31$ species & \\
\hline 15890 & $\mathrm{C}>\mathrm{T}$ & - & $100 \%$ & tRNA-Thr & 1 & $31 / 31$ species & \\
\hline 15939 & $\mathrm{C}>\mathrm{T}$ & - & $7 \%$ & tRNA-Thr & 1 & $13 / 31$ species & \\
\hline \multicolumn{8}{|c|}{ Protein-coding region, unclassified variants } \\
\hline 3308 & $\mathrm{~T}>\mathrm{C}$ & $\mathrm{M}>\mathrm{T}$ & $100 \%^{b}$ & ND1 & 2 & $\mathrm{Hs} / \mathrm{Cf} / \mathrm{Mm} / \mathrm{Rn} / \mathrm{Ag}$ & $0(28)$ \\
\hline 4501 & $\mathrm{C}>\mathrm{T}$ & $S>F$ & $100 \%^{b}$ & ND2 & 1 & $H s / C f$ & $0(20)$ \\
\hline 4561 & $\mathrm{~T}>\mathrm{C}$ & $\mathrm{V}>\mathrm{A}$ & $100 \%^{b}$ & ND2 & 1 & $\mathrm{Hs} / \mathrm{Pt} / \mathrm{Mm} / \mathrm{Rn} / \mathrm{At} / \mathrm{Os}$ & $0(20)$ \\
\hline 5319 & $\mathrm{~A}>\mathrm{G}$ & $\mathrm{T}>\mathrm{A}$ & $100 \%^{b}$ & ND2 & 1 & $H s$ & $0(20)$ \\
\hline 6408 & $\mathrm{~A}>\mathrm{G}$ & $\mathrm{I}>\mathrm{V}$ & $100 \%^{b}$ & $\mathrm{CO} 1$ & 1 & $\mathrm{Hs} / \mathrm{Pt} / \mathrm{Cf} / \mathrm{Mm} / \mathrm{Rn} / \mathrm{Gg} / \mathrm{Dm} / \mathrm{Ag} / \mathrm{Sp}$ & $6(20)$ \\
\hline 7146 & $\mathrm{~A}>\mathrm{G}$ & $\mathrm{T}>\mathrm{A}$ & $100 \%^{b}$ & $\mathrm{CO} 1$ & 2 & $H s / C f$ & $0(28)$ \\
\hline 7389 & $\mathrm{~T}>\mathrm{C}$ & $\mathrm{Y}>\mathrm{H}$ & $100 \%^{b}$ & $\mathrm{CO} 1$ & 2 & $H s / P t$ & $0(28)$ \\
\hline 8516 & $\mathrm{~T}>\mathrm{C}$ & $\mathrm{W}>\mathrm{R}$ & $100 \%^{b}$ & ATP8 & 1 & $\mathrm{Hs} / \mathrm{Pt} / \mathrm{Mm} / \mathrm{Rn}$ & $7(23)$ \\
\hline 8975 & $\mathrm{~T}>\mathrm{C}$ & $\mathrm{L}>\mathrm{P}$ & $100 \%^{b}$ & ATP6 & 1 & $H s / P t / G g$ & $4(20)$ \\
\hline 10680 & $\mathrm{G}>\mathrm{A}$ & $\mathrm{A}>\mathrm{T}$ & $100 \%^{b}$ & ND4L & 1 & $H s / P t / C f / M m / R n$ & $15(28)$ \\
\hline 11447 & $\mathrm{G}>\mathrm{A}$ & $\mathrm{V}>\mathrm{M}$ & $100 \%^{b}$ & ND4 & 1 & $H s / \mathrm{Pt} / \mathrm{Cf}$ & $2(20)$ \\
\hline 13042 & $\mathrm{G}>\mathrm{A}$ & $\mathrm{A}>\mathrm{T}$ & $84 \%$ & ND5 & 1 & $\mathrm{Hs} / \mathrm{Pt} / \mathrm{Cf} / \mathrm{Mm} / \mathrm{Rn} / \mathrm{Gg} / \mathrm{Dm} / \mathrm{Ag} / \mathrm{Ce} / \mathrm{At} / \mathrm{Os}$ & $18(31)$ \\
\hline 13630 & $\mathrm{~A}>\mathrm{G}$ & $\mathrm{T}>\mathrm{A}$ & $100 \%^{b}$ & ND5 & 1 & $\mathrm{Hs} / \mathrm{Pt} / \mathrm{Gg} / \mathrm{Dm} / \mathrm{Ag}$ & $8(28)$ \\
\hline 13880 & $\mathrm{C}>\mathrm{A}$ & $S>Y$ & $100 \%^{b}$ & ND5 & 2 & $H s / P t / A t$ & $0(28)$ \\
\hline 14207 & $\mathrm{G}>\mathrm{A}$ & $\mathrm{T}>\mathrm{I}$ & $100 \%^{b}$ & ND6 & 1 & $H s / \mathrm{Pt} / \mathrm{Cf}$ & $8(28)$ \\
\hline 14258 & $\mathrm{G}>\mathrm{A}$ & $\mathrm{P}>\mathrm{L}$ & $70 \%^{c}$ & ND6 & 1 & $H s / P t$ & $5(20)$ \\
\hline 14766 & $\mathrm{C}>\mathrm{T}$ & $\mathrm{T}>\mathrm{I}$ & $100 \%^{b}$ & CYB & 13 & $H s / P t$ & $8(28)$ \\
\hline 15311 & $\mathrm{~A}>\mathrm{G}$ & $\mathrm{I}>\mathrm{V}$ & $100 \%^{b}$ & CYB & 1 & $\mathrm{Hs} / \mathrm{Pt} / \mathrm{Cf} / \mathrm{Mm} / \mathrm{Rn} / \mathrm{Gg} / \mathrm{Ag} / \mathrm{Ce} / \mathrm{Sp} / \mathrm{Sc} / \mathrm{Kl} / \mathrm{Eg}$ & $1(23)$ \\
\hline 15725 & $\mathrm{C}>\mathrm{T}$ & $\mathrm{L}>\mathrm{F}$ & $100 \%^{b}$ & CYB & 1 & $\mathrm{Hs} / \mathrm{Pt} / \mathrm{Cf} / \mathrm{Gg} / \mathrm{Kl} / \mathrm{Eg} / \mathrm{At}$ & $2(20)$ \\
\hline 15824 & $\mathrm{~A}>\mathrm{G}$ & $\mathrm{T}>\mathrm{A}$ & $100 \%^{b}$ & CYB & 1 & $H s / P t / C f / G g$ & $0(20)$ \\
\hline
\end{tabular}

${ }^{a}$ Mutation loads are determined by mutation specific restriction digestion analysis followed by GeneScan analysis.

${ }^{b}$ Mutations load estimated by MitoChip and/or sequencing results only.

${ }^{c}$ Also confirmed with denaturing high-performance liquid chromatography (dHPLC) analysis.

${ }^{d} \mathrm{Ag}$, Anopheles gambiae; At, Arabidopsis thaliana; Ce, Caenorhabditis elegans; Cf, Canisfamiliaris; Dm, Drosophila melanogaster; Eg, Eremothecium gossypii; Gg, Gallus gallus; Hs, Homo sapiens; Kl, Kluyveromyces lactis; Mm, Mus musculus; Os, Oryza sativa; Pt, Pan troglodytes; Rn, Rattus norvegicus; Sc, Saccharomyces cerevisiae; Sp, Schizosaccharomyces pombe.

${ }^{e}$ The pathogenicity score is calculated according to the criteria set by Mitchell et al. ${ }^{22}$ Since information was not available on all criteria, the maximal score using only the available information is indicated between brackets.

${ }^{\mathrm{h}}$ Detected as heteroplasmic by MitoChip analysis.

tRNA conservations were taken from the website dealing with the compilation of mammalian tRNA genes (http://mamit-trna.u-strasbg.fr/index.html). ${ }^{32}$ 

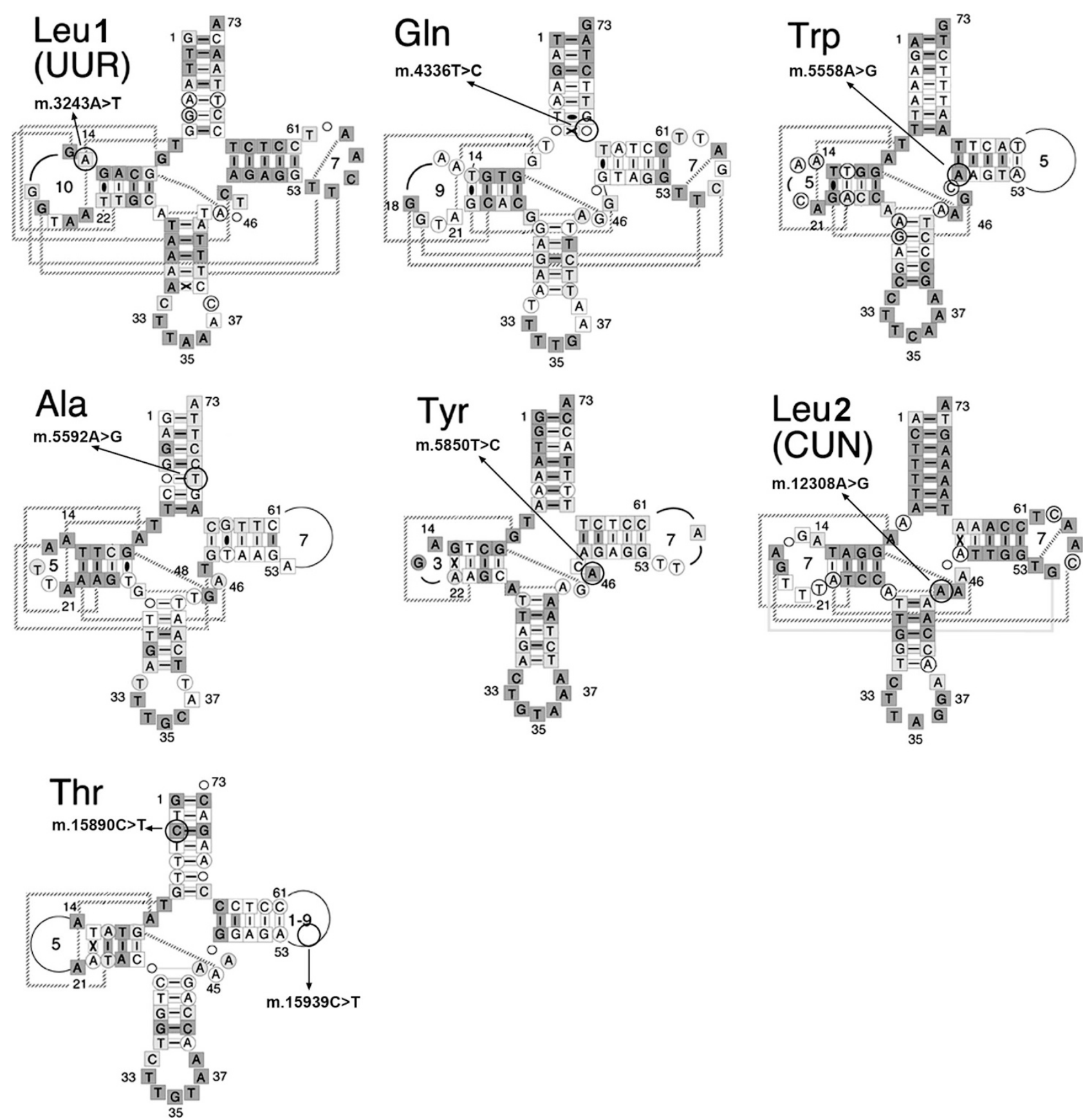

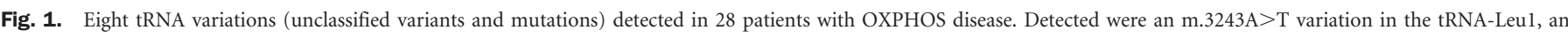

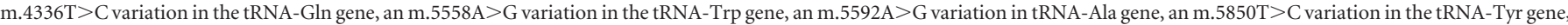

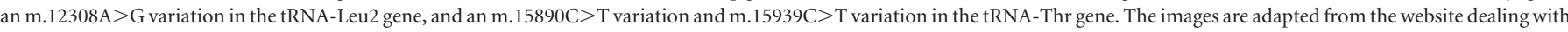
the compilation of mammalian tRNA genes (http://mamit-trna.u-strasbg.fr/index.html). ${ }^{32}$

\section{DISCUSSION}

\section{MitoChip performance and validation}

MitoChip resequencing is a very rapid method to screen the mtDNA for mutations. Eight samples can be analyzed by one technician over a period of 4 days. The average call rate of $94.0 \%$ is within the range described by Affymetrix and others. ${ }^{5}$ The additional $\mathrm{R}$ analysis led to an increase in total base calls and to the identification of 5 UVs (4/7 samples), which would have been missed by GDAS analysis only. Of the 212 chip po- sitions (0.72\%) (134 mtDNA nucleotide positions) which consistently gave a "no call," 197 (124 mtDNA nucleotide positions) were cytosine nucleotides. Previously, it was reported that $1.7 \%$ of the bases always gave a "no call" in a dataset of 26 chips. ${ }^{5}$ About $51 \%$ of these were from regions containing two or more successive $\mathrm{C}$ bases. R-analysis showed that in these regions especially the forward strand differed from the reference sequence, whereas the reverse sequence gave a call equal to the reference sequence. The reason for this is yet unknown. The 
mtDNA nucleotide positions of twelve common mtDNA mutations $(\mathrm{m} .1555 \mathrm{~A}>\mathrm{G}, \mathrm{m} .3243 \mathrm{~A}>\mathrm{G}, \mathrm{m} .3460 \mathrm{G}>\mathrm{A}, \mathrm{m} .8344 \mathrm{~A}>\mathrm{G}$, m.8993T $>$ G/C. m.9176T $>$ C, m.10159T $>$ C, m.11778G $>$ A, m.13513G $>$ A, m.14459G $>$ A, m.14484T $>$ C, and m.14487T $>$ C), were not among the nucleotide positions always giving a "nocall." Within-chip and between-chip reproducibility have already been determined showing a within-chip error rate of $0.0025 \%$ and a between-chip error rate of $0.0027 \%$, illustrating that the base calls were reproducible for $>99.99 \% .{ }^{5}$ In our experiments $97 \%$ of the variations were called correctly in three previously sequenced samples, which is comparable with the $95 \%$ reported before in 18 samples. ${ }^{5}$ In the three test samples, only six variations appeared be false positive after follow-up investigation. It is unlikely that PCR errors are causative for false positive MitoChip calls since there is a large number of templates for the PCR, and the heteroplasmy levels due to sporadic PCR errors will be below the detection limit. As GeneChip Resequencing Arrays only support detection of homozygous and heterozygous single nucleotide substitutions, small deletions ( $5 \mathrm{bp}$ ) could not be detected, although 2 single nucleotide insertions were detected as heteroplasmic "substitutions." One might expect that the heteroplasmic 5-bp deletion would be detected by the MitoChip as a loss of signal, but available software is insufficient for these quantitative analyses, especially if over $50 \%$ of the wild type mtDNA is still present. $^{8}$

Although the GDAS software was designed to detect homozygous (homoplasmic) and heterozygous (50\% heteroplasmy) single base pair substitutions only, it was previously shown that heteroplasmic variations at levels as low as $2 \%$ were detectable. ${ }^{5}$ However, in our dilution experiments only one out of 16 positions could be identified correctly as a heteroplasmic variation in the $5 \%, 10 \%$, and $50 \%$ mix samples. Notably, the number of "no calls" at these 16 heteroplasmic positions was three times higher at the TQT setting of 30 compared to the GDAS default TQT setting of 75, illustrating a difficulty for the algorithm to assign a call for the particular position, probably because of the signal of the heteroplasmic variant. This suggests that further development of the data analysis algorithm and tuning of the data analysis settings can improve the detection level of heteroplasmic mutations. An option would be to customize our R-analysis to make it sensitive for heteroplasmic variations. Nevertheless, we were able to detect a heteroplasmic variation in the tRNA-Thr gene $(\mathrm{m} .15939 \mathrm{C}>\mathrm{T})$ in a patient with a mutation load as low as $7 \%$. Additionally, a pathogenic mutation in the ND5 gene (m.13513G $>$ A) was identified and validated at a heteroplasmic level of 13-15\%. Apparently the sensitivity for heteroplasmy detection is different per position and probably depends on the sequence surrounding the variation.

\section{MitoChip compared to other methods}

Resequencing Chips are powerful if long-range PCR protocols are available and substitutions are the predominant pathogenic mutations, which is the case for the mtDNA. Other methods for mtDNA mutation analysis are conventional sequence analysis, single strand conformation polymorphism
(SSCP) analysis, denaturing gradient gel electrophoresis (DGGE), temperature gradient gel electrophoresis (TGGE), denaturant capillary electrophoresis (DCE) and denaturing high-performance liquid chromatography (dHPLC). ${ }^{13-17}$ These techniques all have their advantages and disadvantages with respect to costs time consumption, high-output possibilities, heteroplasmic detection limit, and type of mutations that can be detected (Table 3). At this moment, heteroduplex-based methods (dHPLC, DGGE, TGGE, and DCE) appear to be more sensitive than the MitoChip for heteroplasmy detection, with detection sensitivities as low as $0.5 \% .{ }^{14-16,18}$ SSCP and conventional sequence analysis are not able to detect low percentages of heteroplasmy. MitoChip resequencing is able to detect heteroplasmic variants at low levels, although the data analysis needs to be optimized to reach similar detection levels. Although the costs per nucleotide are cheaper for conventional sequencing, ${ }^{5}$ considering the overall costs per sample, MitoChip resequencing is preferable over conventional sequencing, mostly due to a tremendous gain in labor costs (8 samples can be analyzed by one technician over a period of 4 days) and automated analysis at comparable bench costs.

\section{MtDNA variations in 28 clinical samples}

The pathogenic m.3243A $>$ T mutation was found in a patient with proximal myopathy, ptosis and ophthalmoplegia at a mutation level of 34\%. Magnetic resonance imaging (MRI) of the brain showed white matter abnormalities and muscle creatine phosphokinase levels were elevated. Muscle biopsy histomorphology revealed ragged red fibers (RRF) and cytochrome c oxidase (COX) negative fibers. This mutation has been previously described in muscle $(81.4 \%)$, skin (69.3\%) and blood (13.8\%) of a 9-year-old girl with muscle weakness, encephalopathy and a reduction of the activity of OXPHOS complexes I, III and IV. ${ }^{19}$ The mutation is located at the same position as the classical MELAS m.A3243A $>\mathrm{G}$ mutation at the first position of the D-loop in the tRNA-Leu1 molecule (Fig. 1), leading to a dramatic loss of aminocylation efficiency. Moreover, residue A14 of this tRNA is strongly conserved

Table 3

Comparision of mtDNA mutation screening methods

\begin{tabular}{|c|c|c|c|c|c|c|c|}
\hline & SSCP & DGGE & TGGE & DCE & dHPLC & Sequencing & MitoChip \\
\hline $\begin{array}{l}\text { Equipment } \\
\text { costs }\end{array}$ & low & low & low & high & high & high & high \\
\hline $\begin{array}{l}\text { Labor } \\
\text { intensive }\end{array}$ & yes & yes & yes & no & no & yes & no \\
\hline High-output & - & - & - & + & + & + & ++ \\
\hline $\begin{array}{l}\text { Heteroplasmy } \\
\text { detection }\end{array}$ & - & ++ & ++ & ++ & ++ & + & $+(+)$ \\
\hline $\begin{array}{c}\text { Homoplasmy } \\
\text { detection }\end{array}$ & + & - & - & - & - & + & + \\
\hline
\end{tabular}

SSCP, single strand conformation polymorphism; DGGE, denaturing gradient gel electrophoresis; TGGE, temperature gradient gel electrophoresis; DCE, denaturant capillary electrophoresis; dHPLC, denaturing high-performance liquid chromatography. 
within all classical tRNA molecules and it is involved in establishing tertiary interactions with other residues of the tRNA. ${ }^{20}$ The pathogenic m.3697G $>$ A mutation in the ND1 gene was detected at a mutation load higher than $97 \%$ in a patient with spastic dystonia, elevated levels of lactate and pyruvate in blood serum and cerebrospinal fluid. The affected amino acid is evolutionary highly conserved (Table 1) and the mutation has been described before in a MELAS patient with $80 \%$ of the m.3697G $>$ A mutation in muscle and $79 \%$ in skin fibroblasts. ${ }^{21}$ Pathogenicity of the mutation was confirmed by fusion experiments with $\rho^{0}$ cell lines (no restoration of complex I activity) combined with blue native polyacrylamide gel electrophoresis (BNPAGE) (low levels of fully assembled complex I). ${ }^{21}$ The m.3697G $>$ A mutations was also evaluated according to the scoring system to assess pathogenicity of complex I mutations. ${ }^{22}$ This score can range from 0 (not pathogenic) to 40 (pathogenic) and is based on several criteria: biochemical defect, functional studies, reports by two or more independent laboratories, heteroplasmy, segregation in the family, and evolutionary conservation. The m.3697G $>$ A mutation reached a score of 32. Our report of this mutation as a second independent laboratory will increase the score to 37 points, which is above the "pathogenic" cutoff of 30. The m.13513A $>$ G mutation was detected in a patient with encephalopathy, strokes, dystonia, signs of Parkinsonism, mental retardation and signs of early fatigued. Biochemical analysis indicated a deficiency of the pyruvate dehydrogenase complex (PDHC) in muscle. The m.13513A $>\mathrm{G}$ transition in the ND5 gene changes an amino acid strongly conserved over 11 species (Table 1) and has been related to MELAS and Leigh syndrome in several reports. ${ }^{23-28}$ The pathogenicity score for this mutation is 39.22 The functional importance of this amino acid is further supported by the finding of a pathogenic mutation m.13514A $>\mathrm{G}$, affecting the same codon, that results in a different amino acid replacement (D393G vs. D393N) in two MELAS patients. ${ }^{29}$

UVs were evaluated with respect to heteroplasmy, evolutionary conservation, functional influence of the effect on tRNA or protein, and segregation in the family. $22,30,31$ Several UVs were likely to be pathogenic, but the effect of others is still unclear. The m.5850T $>\mathrm{C}$ variation had a mutation load of $>98 \%$ in muscle and $13 \%$ in urine, but it could not be detected in blood or hair of the patient. It was not present in blood, hair, or urine from the patient's clinically unaffected mother, brother, and sister. The position of this variation in the tRNA-Tyr gene is evolutionary highly conserved. These data strongly suggest a pathogenic role. A second variation with a high probability of pathogenicity was the homoplasmic m.3229-3230insA. This variation is located in the transcription terminator site and may disturb the function of this site. Moreover, the location of this variation being exactly between the $16 \mathrm{~S}$ rRNA gene and the tRNALeu1 gene might result in a faulty cleavage of the RNA after transcription. A faulty cleavage could have consequences for the function of the tRNA-Leu1 and 16S rRNA genes. The m.13042G>A variation in the ND5 gene was heteroplasmic (84\%) and the involved amino acid was also strongly conserved (Table 2), which favors a pathogenic role. The $\mathrm{m} .14258 \mathrm{G}>\mathrm{A}$ variation in the ND6 gene was also a heteroplasmic ( $70 \%$ ) variation in a protein coding gene. Although this variation is heteroplasmic, the affected amino acid is not well conserved. The m.15939C $>\mathrm{T}$ variation in the tRNA-Thr gene was also heteroplasmic (7\%) in the patient's muscle. The variation is located in the T-loop of the tRNA molecule and not involved in establishing tertiary interactions in the tRNA molecule, making a pathogenic role less likely. The other homoplasmic variations (Table 2) have to be further evaluated, as it is difficult to determine pathogenicity solely based on criteria like heteroplasmy and conservation alone. Functional and family studies will be performed to provide the definite evidence.

Resequencing does not only detect known pathogenic mutations or polymorphisms, but also UVs and risk factors for unrelated pathology, like cancer, Alzheimer's disease or Parkinson's disease. As the significance of this latter group of variations for individual cases and their families is yet unclear and the risk factors can not explain the primary pathology, it is evident that these data have to be carefully dealt with by the clinicians. Patients should be counseled about the uncertainties of some of the observations and the difficulties to interpret part of the results. However, this is inevitable and does not counterweight the genetic diagnoses made. It will also be a temporary problem, as due to the joint sequencing efforts more and more information will become available about neutral polymorphisms, genuine risk factors of disease, and actual pathogenic mutations.

\section{CONCLUSION}

MitoChip resequencing is a fast, cost-effective and reliable method with high-output capabilities for complete mtDNA screening. A quarter of the patients with OXPHOS disease can be genetically diagnosed by this technique, based on the detection of three pathogenic and three or four likely pathogenic mutations in 28 patients, which confirms the percentage previously described. ${ }^{3}$ Because of the growing number of mtDNA mutations combined with the increasing clinical heterogeneity, MitoChip resequencing is the preferred method to screen the mtDNA for mutations, especially when symptom specific screening and screening of only the most common mtDNA mutations falls short.

\section{ACKNOWLEDGMENTS}

This research was supported by the Princess Beatrix Foundation, (Grant number: MAR99-0111) and the MitoCircle project (EU grant, Sixth Framework Program, contr. no. 005260).

\section{References}

1. Chinnery PF. New approaches to the treatment of mitochondrial disorders. Reprod Biomed Online 2004;8:16-23.

2. Wallace DC. Mitochondrial diseases in man and mouse. Science 1999;283:14821488 .

3. Thorburn DR. Mitochondrial disorders: prevalence, myths and advances. J Inherit Metab Dis 2004;27:349-362.

4. Brandon MC, Lott MT, Nguyen KC, Spolim S, et al. MITOMAP: a human mitochondrial genome database-2004 update. Nucleic Acids Res 2005;33:D611-D613.

5. Maitra A, Cohen Y, Gillespie SE, Mambo E, et al. The Human MitoChip: a highoutput sequencing microarray for mitochondrial mutation detection. Genome Res 2004;14:812-819. 
6. Jakupciak JP, Wang W, Markowitz ME, Ally D, et al. Mitochondrial DNA as a cancer biomarker. J Mol Diagn 2005;7:258-267.

7. Mullenbach R, Lagoda PJ, Welter C. An efficient salt-chloroform extraction of DNA from blood and tissues. Trends Genet 1989;5:391.

8. Comi GP, Bordoni A, Salani S, Franceschina L, et al. Cytochrome c oxidase subunit I microdeletion in a patient with motor neuron disease. Ann Neurol 1998;43:110-116.

9. Pease AC, Solas D, Sullivan EJ, Cronin MT, et al. Light-generated oligonucleotide arrays for rapid DNA sequence analysis. Proc Natl Acad Sci U S A 1994;91:5022-5026.

10. Lipshutz RJ, Fodor SP, Gingeras TR, Lockhart DJ. High density synthetic oligonucleotide arrays. Nat Genet 1999;21:20-24.

11. Andrews RM, Kubacka I, Chinnery PF, Lightowlers RN, et al. Reanalysis and revision of the Cambridge reference sequence for human mitochondrial DNA. Nat Genet 1999;23:147.

12. R Development Core Team. R: A language and environment for statistical computing. 2004. Vienna, Austria: R Foundation for Statistical Computing.

13. Suomalainen A, Ciafaloni E, Koga Y, Peltonen L, et al. Use of single strand conformation polymorphism analysis to detect point mutations in human mitochondrial DNA. J Neurol Sci 1992;111:222-226.

14. van Orsouw NJ, Zhang X, Wei JY, Johns DR, et al. Mutational scanning of mitochondrial DNA by two-dimensional electrophoresis. Genomics 1998;52:27-36.

15. van den Bosch BJ, de Coo RF, Scholte HR, Nijland JG, et al. Mutation analysis of the entire mitochondrial genome using denaturing high performance liquid chromatography. Nucleic Acids Res 2000;28:E89.

16. Bjorheim J, Ekstrom PO. Review of denaturant capillary electrophoresis in DNA variation analysis. Electrophoresis 2005;26:2520-2530.

17. Wartell RM, Hosseini SH, Moran CP. Detecting base pair substitutions in DNA fragments by temperature-gradient gel electrophoresis. Nucleic Acids Res 1990;18: 2699-2705.

18. Wong LJ, Boles RG. Mitochondrial DNA analysis in clinical laboratory diagnostics. Clin Chim Acta 2005;354:1-20.

19. Shaag A, Saada A, Steinberg A, Navon P, et al. Mitochondrial encephalomyopathy associated with a novel mutation in the mitochondrial tRNA(leu)(UUR) gene (A3243T). Biochem Biophys Res Commun 1997;233:637-639.

20. Sohm B, Frugier M, Brule H, Olszak K, et al. Towards understanding human mitochondrial leucine aminoacylation identity. J Mol Biol 2003;328:995-1010.
21. Kirby DM, McFarland R, Ohtake A, Dunning C, et al. Mutations of the mitochondrial ND1 gene as a cause of MELAS. J Med Genet 2004;41:784-789.

22. Mitchell AL, Elson JL, Howell N, Taylor RW, et al. Sequence Variation In Mitochondrial Complex I Genes: Mutation Or Polymorphism? J Med Genet 2006;43:175-179.

23. Pulkes T, Eunson L, Patterson V, Siddiqui A, et al. The mitochondrial DNA G13513A transition in ND5 is associated with a LHON/MELAS overlap syndrome and may be a frequent cause of MELAS. Ann Neurol 1999;46:916-919.

24. Sudo A, Honzawa S, Nonaka I, Goto Y. Leigh syndrome caused by mitochondrial DNA G13513A mutation: frequency and clinical features in Japan. J Hum Genet 2004;49:92-96.

25. Kirby DM, Boneh A, Chow CW, Ohtake A, et al. Low mutant load of mitochondrial DNA G13513A mutation can cause Leigh's disease. Ann Neurol 2003;54: 473-478.

26. Santorelli FM, Tanji K, Kulikova R, Shanske S, et al. Identification of a novel mutation in the mtDNA ND5 gene associated with MELAS. Biochem Biophys Res Commun 1997;238:326-328.

27. Chol M, Lebon S, Benit P, Chretien D, et al. The mitochondrial DNA G13513A MELAS mutation in the NADH dehydrogenase 5 gene is a frequent cause of Leighlike syndrome with isolated complex I deficiency. J Med Genet 2003;40:188-191.

28. Penisson-Besnier I, Reynier P, Asfar P, Douay O, et al. Recurrent brain hematomas in MELAS associated with an ND5 gene mitochondrial mutation. Neurology 2000; 55:317-318.

29. Corona P, Antozzi C, Carrara F, D'Incerti L, et al. A novel mtDNA mutation in the ND5 subunit of complex I in two MELAS patients. Ann Neurol 2001;49:106-110.

30. DiMauro S, Schon EA. Mitochondrial DNA mutations in human disease. Am J Med Genet 2001;106:18-26.

31. McFarland R, Elson JL, Taylor RW, Howell N, et al. Assigning pathogenicity to mitochondrial tRNA mutations: when "definitely maybe" is not good enough. Trends Genet 2004;20:591-596.

32. Helm M, Brule H, Friede D, Giege R, et al. Search for characteristic structural features of mammalian mitochondrial tRNAs. Rna 2000;6:1356-1379.

33. Ingman M, Gyllensten U. mtDB: Human Mitochondrial Genome Database, a resource for population genetics and medical sciences. Nucleic Acids Res 2006;34: D749-751. 